\title{
Estudio de las mariposas en la Institución Educativa Las Damas del municipio de Calamar (Guaviare, Colombia), una estrategia en la búsqueda de conciencia ambiental.
}

\author{
Study of butterflies at Institución educativa Las Damas of \\ Calamar Municipality (Guaviare, Colombia), a strategy in \\ search of environmental awareness.
}

Por: * Arenas Jaramillo Johan Gustavo, ** Moreno Niño Anaya Carolina, *** Giraldo Gustavo ${ }^{1}$

\begin{tabular}{|l|}
\hline Recibido: 19-12-2008 \\
\hline Aceptado: 04-05-2009 \\
\hline
\end{tabular}

Resumen: Este trabajo buscó generar una Conciencia Ambiental que mejorara las relaciones de los integrantes de la comunidad con su entorno, propiciando actitudes de responsabilidad, respeto y conservación del ambiente. Para ello, se propuso el estudio y cría de mariposas como estrategia en la Educación Ambiental de la escuela. Se utilizó la metodología de trabajo denominada cartografía social para identificar la forma en que los estudiantes se apropiaban de su entorno y se relacionaban con las mariposas. Durante el desarrollo de este estudio se logró poner en marcha procesos organizativos generando una dinámica de trabajo que posibilitó la sensibilización y constante participación de los diferentes actores que conforman la comunidad. Se realizaron salidas de campo las cuales fueron utilizadas como escenarios de aprendizaje donde se realizaron procesos de análisis y reflexión permitiendo a los niños, niñas y docentes reconocerse como actores principales, responsables de la transformación de su entorno, involucrando una mirada sistémica (cultural, social, política y económica).

Palabras Claves: Conciencia Ambiental, Cartografía Social, Mariposas.

Abstract: This work sought to generate an Environmental awareness, which improved the relationships of the members of the community with the environment, favouring attitudes of responsibility, respect and conservation of the environment. For this, the the study and the Breeding of butterflies was proposed as strategy in the Environmental Education of the school. The work methodology that was used is called social cartography to identify the form how the students were appropriating their environment and they related to

\footnotetext{
$1{ }^{*}$ Licenciado en Biología Universidad Distrital Francisco José de Caldas. Asistente Cátedra UNESCO en: desarrollo del niño Universidad Distrital Francisco José de Caldas. johanbiology@hotmail.com

** Licenciada en Biología Universidad Distrital Francisco José de Caldas.

*** Profesor proyecto Curricular de Licenciatura en Biología Universidad Distrital Francisco José de Caldas. ggiraldoq@udistrital.edu.co.

Integrantes Grupo de Investigación en Calidad Ambiental.
} 
butterflies. During the development of this study it was achieved to start organizational processes generating a dynamics of work that made possible the sensitization and constant participation of the different actors who make up the community. The field trips were carried out, which were used like learning scenes where processes of analysis and reflection were mad, allowing the children and teachers recognized themselves as principal actors, responsible for the transformation of their environment, involving a cultural, social, political and economic systemic look.

Keys words: Environmental awareness, Social Cartography, Butterflies.

\section{INTRODUCCIÓN}

En Colombia se han realizado diferentes esfuerzos para incluir la dimensión ambiental en el currículo a partir de proyectos y actividades que permiten integrar las diversas áreas del conocimiento en la búsqueda de soluciones a problemáticas particulares. Los Proyectos Ambientales Escolares (PRAES) incorporan la problemática ambiental local al quehacer de las instituciones educativas, teniendo en cuenta la dinámica natural y sociocultural del contexto (MEN, 2002). Mediante esta experiencia se buscó contribuir al fortalecimiento del proceso que se venía desarrollando en la escuela en torno al PRAE, para ello se propusieron un conjunto de actividades relacionadas con el estudio y la cría de mariposas, ya que éstas son un grupo de insectos ideal para incentivar la participación comunitaria así como sensibilizar a las comunidades acerca de la importancia de cuidar y conservar los ecosistemas, puesto que son llamativas, tienen ciclos vitales cortos, son fáciles de criar y además poseen alta especificidad hacia las plantas de las cuales se alimentan en estado de oruga. Se utilizó la cartografía social la cual es una metodología alternativa que permite a las comunidades conocer y construir un conocimiento integral de su territorio para que puedan elegir una mejor manera de vivirlo (Habegger y Mancila, 2006). Ésta es una forma de investigación humanista y humanizadora, así como una propuesta conceptual novedosa que hace uso de instrumentos técnicos y vivenciales a través de la construcción simbólica del territorio (entendido éste tanto por el espacio como por las relaciones que interactúan en y sobre él), de esta forma se abren perspectivas para una mejor comprensión de la realidad territorial. Con la cartografía social se buscó identificar la forma como la comunidad se apropiaba de su territorio y explorar algunos saberes y creencias relacionadas con las mariposas. Es importante recalcar que la cartografía social permite la construcción de conocimiento colectivo, donde se utilizan los mapas como centro de motivación reflexión y redescubrimiento del territorio.

La Institución Educativa Las Damas (I.E.L.D), presta sus servicios principalmente a la comunidad de la vereda Las Damas cuya economía se basa en los cultivos ilícitos, monocultivos y la ganadería extensiva. Estas formas de explotación de los recursos han generado degradación de los suelos, fragmentación de los bosques (praderización), contaminación de las fuentes de agua, principalmente del Nacedero la Cristalina, recurso Hídrico de la población, trayendo como consecuencia la disminución de especies nativas (PRAE I.E.L.D. 2007)²; problemáticas ambientales que afectan a la comunidad; ya que

2 Este análisis se basa en el diagnóstico de la situación ambiental de las Damas que se encuentra en el PRAE de la I.E.L.D titulado “Protección y conservación del Nacedero La Cristalina" 2007 
deterioran su hábitat, disminuyendo la posibilidad de aprovechar y utilizar de manera razonable los recursos que el ecosistema provee. Con este estudio se buscó generar en la comunidad una conciencia ambiental que permitiera mejorar las relaciones de los individuos con el medio, generando actitudes de responsabilidad, respeto y conservación del entorno.

\section{Situación ambiental de las Damas}

El siguiente análisis permite contextualizar la situación ambiental de la vereda Las Damas desde una visión sistémica, ya que tiene en cuenta las variables sociedad, espacio, población y recursos poniendo en evidencia la dinámica ambiental que ocurre allí:

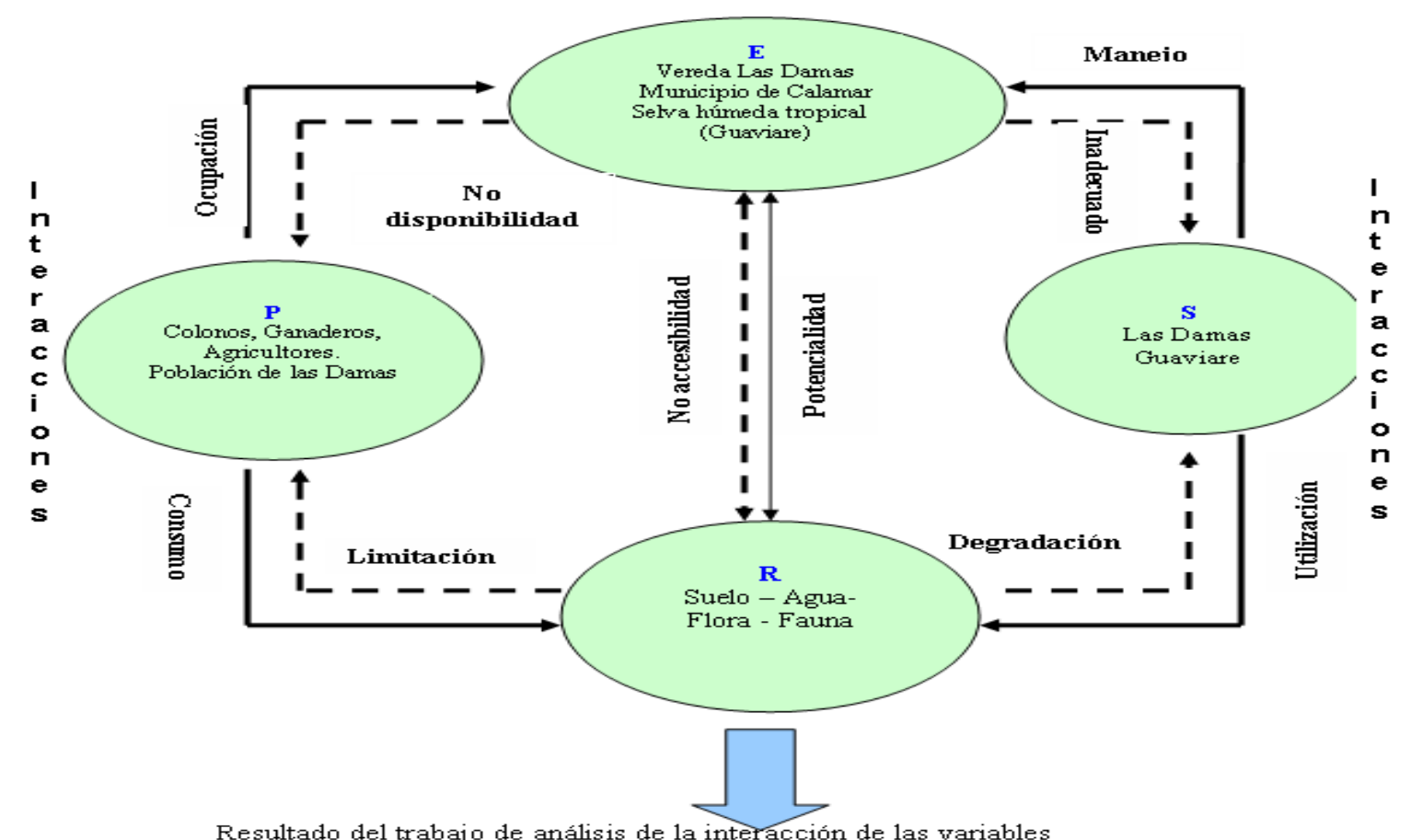

Resultado del trabajo de análisis de la interacción de las variables

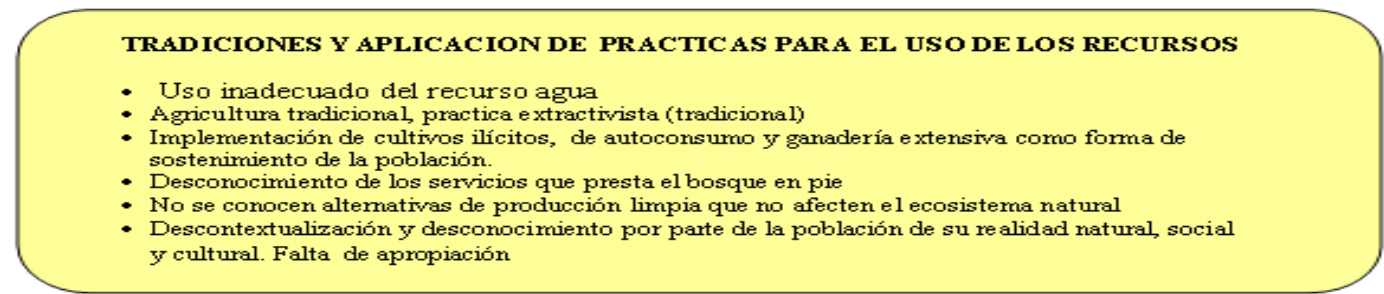

Fig. 1. Basado en el esquema de Goffin (1992)(MEN, 2002) Representa a la Sociedad (S), los recursos $(\boldsymbol{R})$, la población $(\boldsymbol{P})$, y el espacio $(\boldsymbol{E})$, como componentes fundamentales del sistema ambiental; los cuales se encuentran en constante interacción, que mediadas en el tiempo, dan cuenta de la dinámica del mismo. Estas interacciones entre los $R$, la $S$, la P y el E; pueden ser de, degradación (retroacción negativa), utilización (interacción). El cuadro se encuentra contextualizado a la realidad de las Damas.

En el esquema, las flechas continuas indican la interacción que se da entre diferentes componentes del sistema ambiental de la vereda Recursos (R), Población (P), Sociedad (S) 
y Espacio (E), mientras que las flechas discontinuas indican una retroacción negativa consecuencia de la interacción entre éstos, es así como en la vereda Las Damas (Guaviare) la utilización continua de los recursos por parte de la comunidad han generado la degradación del ecosistema, causando fragmentación de los bosques, contaminación de las fuentes de agua y disminución de especies nativas; problemáticas ambientales que deterioran el hábitat, limitando la disponibilidad de recursos. La sociedad de las Damas no ha incorporado actitudes y acciones necesarias para cuidar, proteger y conservar el entorno, producto de una larga tradición de explotación rural del territorio en parte debido a la falta de una Conciencia Ambiental, tampoco se han generado alternativas de producción que implementen tecnologías limpias; lo cual permitiría la recuperación del ecosistema de la selva húmeda tropical. Las actitudes, comportamientos y sistemas de explotación de los recursos, implementados por varias generaciones de colonos, ganaderos y agricultores han dado lugar a su limitación y degradación, disminuyendo la potencialidad del ecosistema, también han causado deterioro del espacio y de la calidad de vida de sus habitantes.

\section{MATERIALES Y MÉTODOS}

La presente investigación se realizó en la Institución Educativa Las Damas I.E.L.D, sede principal de un complejo de centros educativos. Ubicada en la Vereda Las Damas a $14 \mathrm{Km}$ al norte del Municipio de Calamar, y a $58 \mathrm{Km}$ al sur de San José del Guaviare, la temperatura oscila entre 28 a $35^{\circ} \mathrm{C}$, dependiendo de la época se destacan dos periodos anuales, uno con fuertes lluvias de marzo a noviembre y otro de sequía de diciembre a febrero, el clima es de tipo estacional cálido. El Guaviare se encuentra ubicado en el sur oriente del país, dentro de la región de la Amazonía, es una zona de gran interés biológico por la variedad de especies de flora y fauna, resultante de la confluencia de diversos elementos florísticos de los biomas Amazonense y Orinocense.

La investigación se dividió en tres fases: 1. Conocimiento y acercamiento de la comunidad, 2. Estudio, observación y establecimiento de sistemas de cría de mariposas y 3. Fortalecimiento del Comité Ambiental de la institución. Estos tres procesos enfocados hacia el estudio de las mariposas se realizaron simultáneamente y estuvieron articulados entre sí. Con el fin de explorar la forma como la comunidad se apropia de su entorno se realizaron salidas de campo a algunos sitios cercanos a la escuela y posteriormente talleres con los niños de primaria en los cuales se crearon mapas veredales que permitieron ubicar los lugares considerados por los estudiantes como los más representativos. Por otro lado, para indagar algunas creencias sobre mariposas y la relación de la comunidad con éstas se realizaron entrevistas y talleres a los niños de primaria y algunos padres de familia, posteriormente la información proporcionada fue categorizada y consignada en una matriz de registro para ser analizada, el marco conceptual de las categorías que sustentan la matriz donde se consigno la información se comprende desde la siguiente jerarquía: 


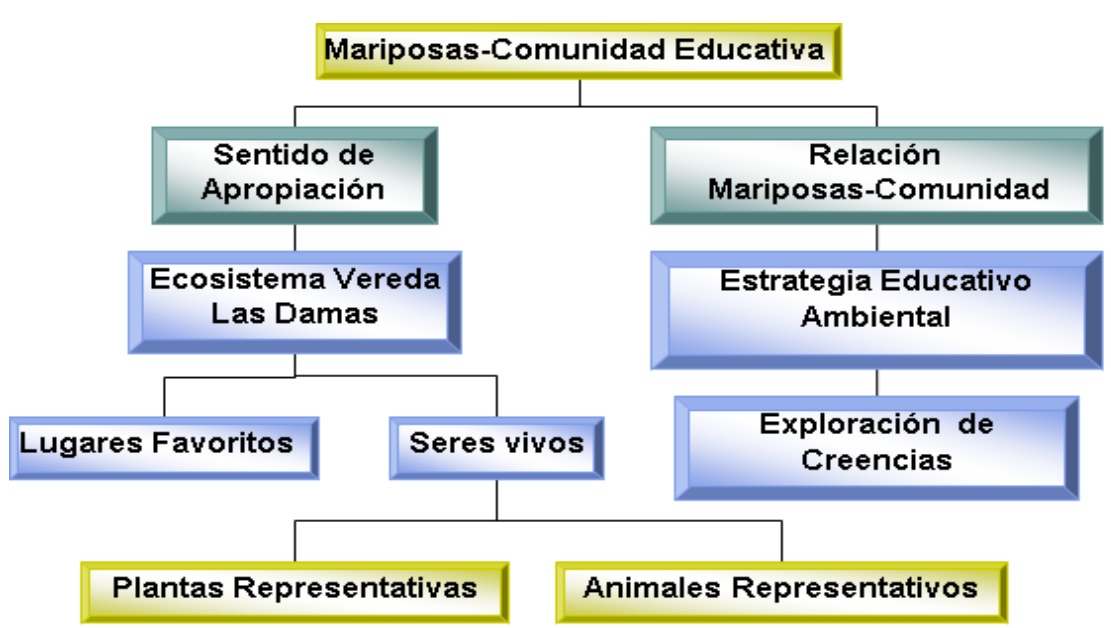

Fig. 2 Mapa conceptual que muestra las categorías analizadas mediante la cartografía social.

Durante la segunda fase se dio inicio a la cría de mariposas en la escuela , para lo cual se implementaron diferentes sistemas de cría, el primer sistema implementado se denominó rancheo, que consiste en que las mariposas ovopositan in situ en sus respectivas plantas hospederas donde el productor recolecta los huevos y las orugas luego son criadas en cautiverio (Gómez, 2006), para ello se realizaron salidas de campo en donde se colectaron orugas que fueron posteriormente criadas en la escuela, los estudiantes cuidaron, alimentaron y realizaron el seguimiento a los cambios que presentaban durante su periodo de desarrollo. Para facilitar el proceso de colecta y cría, se realizaron talleres y se utilizaron guías de campo elaboradas por el equipo investigador, donde se especificaban los procedimientos necesarios para el cuidado y alimentación de las orugas, así como las características biológicas de las mariposas. El segundo sistema utilizado fue la cría ex situ para lo cual se construyó un vivario con un área de $6 \mathrm{~m}^{2}$ y una altura de $2.50 \mathrm{~m}$ (ver Fig. 3), para su construcción se utilizaron tubos de PVC de 2, 2.50 y de 3 metros, polisombra, hilo y nylon; en su interior fueron sembradas plantas hospederas (plantas nutricias de las orugas) y plantas nectaríferas (plantas con flores) para garantizar la alimentación de los adultos, adicionalmente se colocaron nectarios artificiales en los cuales se dispuso azúcar, estiércol, frutas en descomposición y agua. El vivario fue ubicado en un lugar especial dentro de la escuela el cual fue llamado el Jardín de las Mariposas, en este sitio también fueron sembradas plantas hospederas y nectaríferas, para asegurar la visita constante de mariposas y la obtención de posturas de las mismas. Con el fin de crear un catalogo de mariposas se realizaron observaciones y muestreos durante los meses de agosto, septiembre y octubre del 2007 en algunos sitios específicos de la vereda como el Nacedero la Cristalina, Caño Bálsamo, La escuela, y Cuatro caminos, posteriormente las mariposas colectadas fueron identificadas y comparadas con el material que se encuentran en la colección de mariposas SCHMIDT-MUMM del Instituto Alexander Von Humboldt. La última etapa del proceso consistió en fortalecer el comité ambiental de la escuela para lo cual se realizaron diferentes sesiones de trabajo con los integrantes donde se definieron los objetivos, propósitos y actividades a realizar con el fin de dinamizar el proceso educativo ambiental de la escuela. 

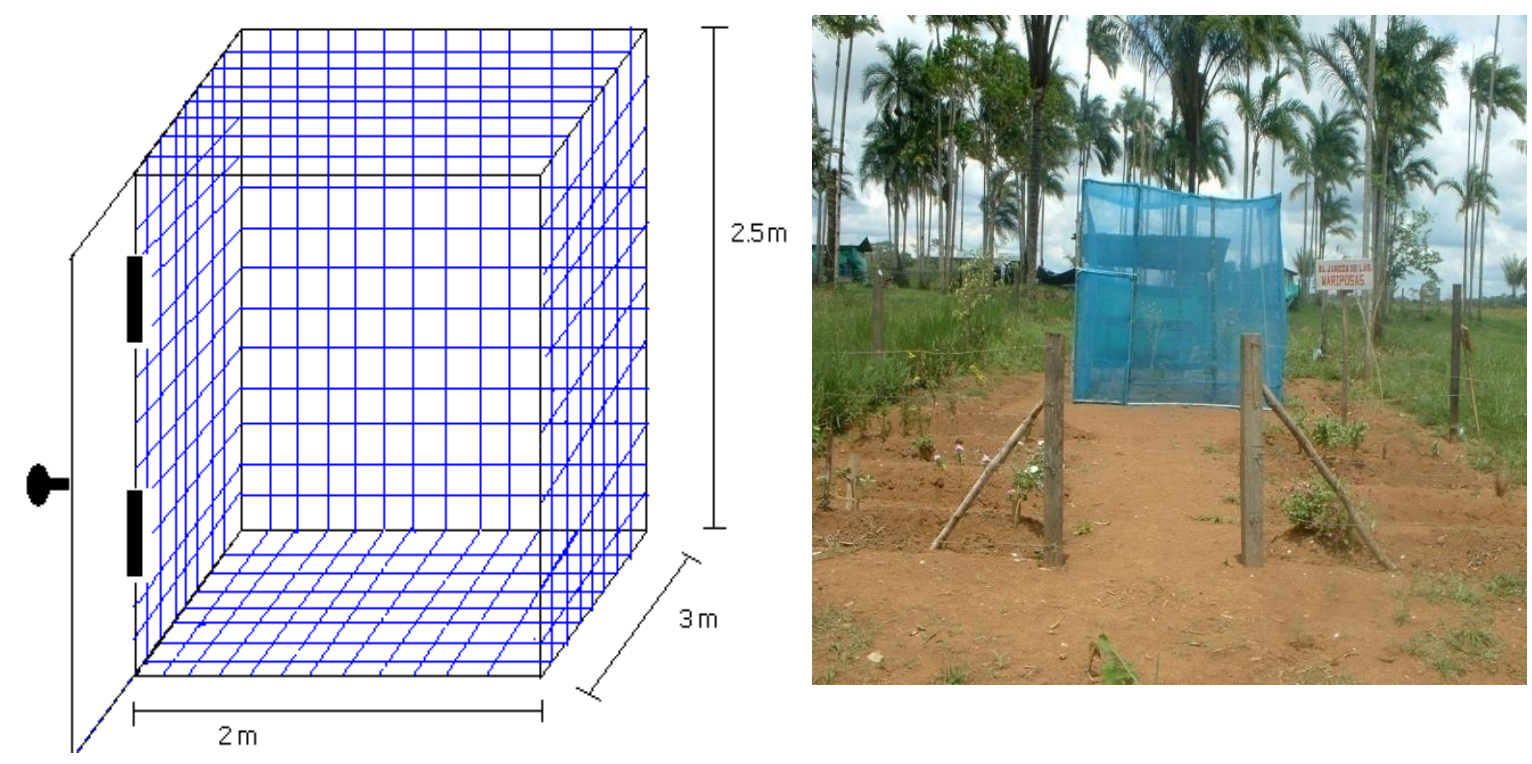

Fig. 3 Estructura del vivario construido para la cría de mariposas.

\section{RESULTADOS Y DISCUSIÓN}

Durante los talleres realizados con los estudiantes de primaria se les pidió que hicieran un dibujo y en él plasmaran los lugares de su preferencia, la mayoría referenció la escuela, la casa y el nacedero la Cristalina (ver Fig. 4), se pudo analizar que los niños y niñas prefieren los sitios cercanos, cotidianos, en los cuales construyen su identidad, puesto que "la construcción de identidad es fundamental en el proceso de configuración de territorios" (Rodríguez, 1998 p.253), entendiendo la identidad como un proceso donde el individuo se concibe a sí mismo en relación con el otro, tanto a través de signos como de diferenciación y de reconocimiento.

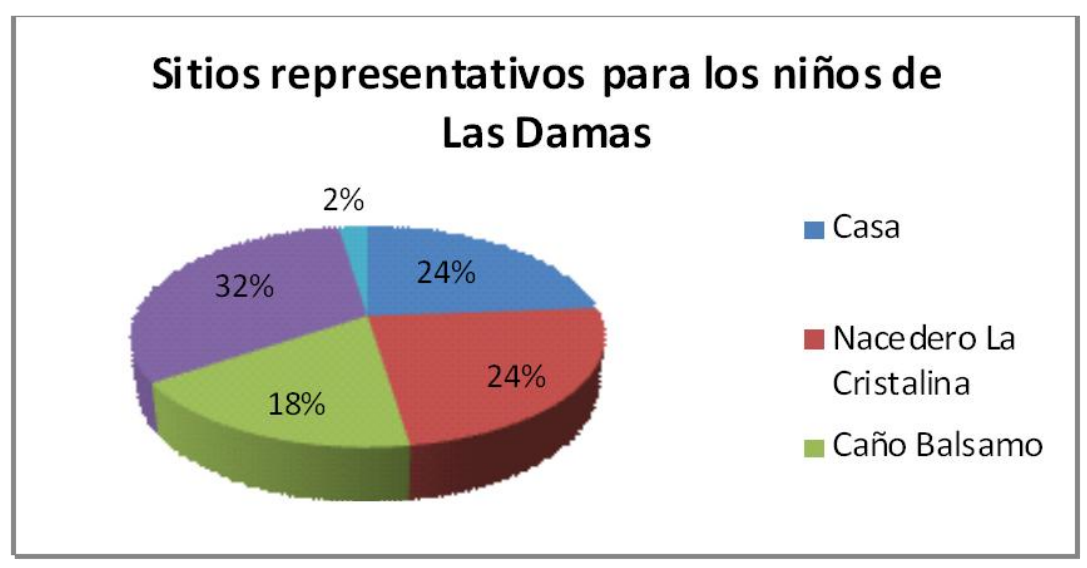

Fig 4. Muestra la relación en porcentajes de acuerdo al número de niños que referenciaron estos sitios en los mapas veredales. 
Tabla 1. Sitios reconocidos por los niños

\begin{tabular}{|c|c|}
\hline Sitio representativo & Explicación de su importancia \\
\hline La Casa & $\begin{array}{l}\text { yar de hábitat, allí los niños se desarrollan en un } \\
\text { de familia, se generan valores y costumbres } \\
\text { del núcleo familiar }\end{array}$ \\
\hline La escuela & $\begin{array}{l}\text { par de socialización por excelencia, allí los niños } \\
\text { en relaciones de intercambio y de reconocimiento } \\
\text { smos }\end{array}$ \\
\hline Nacedero La cristalina & $\begin{array}{l}\text { ar es muy importante para la comunidad, ya que } \\
\text { itio los habitantes del caserío y la escuela se } \\
\text { n de agua, el PRAE de Las Damas gira en torno a } \\
\text { federo. }\end{array}$ \\
\hline Caño Bálsamo & $\begin{array}{l}\text { gar representativo para los niños que viven cerca } \\
\text { sun sitio de diversión para ello y ellas. }\end{array}$ \\
\hline Rio Unilla & $\begin{array}{l}\text { más cercano, a la escuela allá van a pescar, cazar } \\
\text { as personas tienen sembrados cerca al rio. }\end{array}$ \\
\hline
\end{tabular}

Los lugares representados por los niños y niñas en los mapas, son aquellos que han generado en ellos y ellas una identidad territorial pero más allá de eso, una identidad cultural, puesto que ésta encierra un sentido de pertenencia a un grupo social con el que se comparten rasgos culturales, costumbres, valores y creencias que se pueden ver claramente reflejados en las características que hacen que estos sitios sean representativos. La identidad es el sentido de pertenencia a una colectividad, a un sector social, a un grupo específico de referencia. Esta colectividad puede estar por lo general localizada geográficamente, pero no de manera necesaria. La identidad territorial es dinámica. Las personas identifican un territorio y se identifican con él. Este proceso se apoya en elementos variados como naturaleza, paisaje, medioambiente construido, la cultura y etnicidad, el éxito económico, pobreza, fronteras entre él, nosotros y ellos, historias, utopías, etc. Los lugares relacionados por los niños en los dibujos que realizaron son: la casa, la escuela, el Nacedero la Cristalina, Caño Bálsamo, el puerto, el río Unilla. Las características de cada uno de estos sitios que explican su importancia para los niños de Las Damas se pueden observar en la tabla 1.

En la búsqueda de conocimientos o creencias sobre mariposas se realizaron encuestas a los niños y algunos padres de familia, en las cuales se indagaba sobre su ciclo de vida, también se preguntó sobre historias o algunas creencias que tuvieran que ver con las mariposas, su origen, sus transformaciones, encontrado que las mariposas para la comunidad de Las Damas pueden simbolizar muchas cualidades, convertirse en emblemas, a veces representan una desgracia ó una ilusión, imaginarios que finalmente 
se transforman en conocimiento, que al pasar de generación en generación da origen a creencias. Como lo dice Villegas (citado en Vélez y Salazar, 1991), las mariposas además de ser animales vistosos, graciosos y representativos de la naturaleza, han sobrevolado la imaginación del hombre (ver tabla 2).

Tabla 2. Algunas creencias encontradas acerca de las mariposas en los habitantes de la vereda Las Damas, municipio Calamar (Guaviare, Colombia)

\begin{tabular}{|c|c|c|}
\hline $\begin{array}{l}\text { Tema } \\
\text { central }\end{array}$ & Respuestas encontradas en la comunidad & $\begin{array}{c}\text { Preguntas } \\
\text { que } \\
\text { orientaron la } \\
\text { investigación }\end{array}$ \\
\hline $\begin{array}{c}\text { Historias } \\
\text { o } \\
\text { creencias } \\
\text { sobre } \\
\text { mariposas }\end{array}$ & $\begin{array}{l}\text {-Que las mariposas son venenosas por que sueltan un } \\
\text { polvito-. -Que algunas mariposas son de mala suerte, } \\
\text { generalmente las que son de color negro-. } \\
\text {-Las alas de las mariposas son buenísimas para la piel, solo } \\
\text { se deben machacar las alas, y lo que le quede se lo coloca en } \\
\text { la cara-. } \\
\text {-Los gusanos adivinadores (Pupas); jadivinador adivinador } \\
\text { hacia donde queda Calamar-i (existe la creencia en la } \\
\text { comunidad de que a las pupas se les puede realizar } \\
\text { alguna pregunta y ellas pueden responder moviendo } \\
\text { su abdomen). } \\
\text { Una vez cuando me levante, lo primero que observe pegado } \\
\text { en el marco de la puerta fue una mariposa, grande y negra; } \\
\text { mi marido me dijo: eso es que alguien se va a morir, y fijo se } \\
\text { murió un vecino-; }\end{array}$ & $\begin{array}{c}\text { ¿Conoce } \\
\text { alguna } \\
\text { historia o } \\
\text { creencia } \\
\text { relacionada } \\
\text { con las } \\
\text { mariposas? }\end{array}$ \\
\hline Orugas & $\begin{array}{l}\text {-Nunca creímos que las mariposas salgan de los gusanos } \\
\text { (Orugas). } \\
\text {-Nosotros matamos todos los gusanos porque son una plaga } \\
\text { que se come las plantas-. } \\
\text {-El gusano polla que se alimenta de la coca no se transforma } \\
\text { en mariposa-. } \\
\text {-Que los gusanos de la coca, eran una plaga que el gobierno } \\
\text { había soltado en las avionetas, para que se comiera los }\end{array}$ & $\begin{array}{c}\text { ¿Qué piensa } \\
\text { con respecto a } \\
\text { las orugas? } \\
\text { ¿Están } \\
\text { relacionadas } \\
\text { con el origen } \\
\text { de las } \\
\text { mariposas? }\end{array}$ \\
\hline
\end{tabular}




\begin{tabular}{|l|l|l|}
\hline cultivos-. & \\
-Las mariposas salen de las plantas- & \\
& & \\
\hline
\end{tabular}

La exploración de estas creencias permitió realizar un análisis de los conocimientos que los niños, niñas y algunos padres de familia tenían acerca de las mariposas, de esta manera se tuvo un punto de referencia que guiara la acciones a realizar durante la implementación de los sistemas de cría. Se indagó cual era el origen de las mariposas, encontrando en la mayoría de entrevistados respuestas como, -Las mariposas salen de las plantas-, -Nunca creímos que las mariposas salieran de los gusanos-; lo cual evidenció un desconocimiento en cuanto al ciclo de vida y por ende a su procedencia, los habitantes no relacionaban las orugas (gusano), ni las pupas (organismos muy habituales en su entorno) con el origen de las mariposas, basados en argumentos como que no creían que de orugas desagradables y algunas peligrosas, que se comían las plantas que con tanto esmero cuidaban, podían emerger mariposas. Esta exploración de creencias permitió incentivar la búsqueda de conocimiento en los padres y estudiantes generando curiosidad e interés hacia la cría de mariposas. Durante la implementación de los sistemas de cría las plantas hospederas sembradas tanto en el Jardín de las Mariposas como en el interior del vivario fueron Limón (Citrus limón) y Maracuyá (Passiflora edulis). El limón que pertenece a la familia Rutaceae es la planta hospedera de Heraclides anchisiades anchisiades (Papilionidae) mariposa criada exitosamente en la escuela (Fig. 8); sus larvas son de color marrón oscuras (Fig. 5), e imitan el excremento de las aves (Lecrom, 2002). Es importante resaltar la sincronización en cuanto al tiempo de empupado de las orugas y su posterior eclosión, ya que como característica de esta especie sus orugas empupan y eclosionan al mismo tiempo, lo cual pudo evidenciarse durante su cría. Las crisálidas (Fig. 7) tiene una gran semejanza con una ramita seca, la región anal es puntiaguda y la cefálica roma, con una corta proyección de la parte ventral. "Ésta se sujeta al tronco o ramas de la planta hospedera por la región anal cabeza arriba y se encuentra rodeada por una fibra de seda que la mantiene inclinada en un ángulo de 45o "(Álvarez, Alvarez, 1984, p 94). El ciclo completo de la mariposa dura aproximadamente 49 días pasando por huevo, oruga, crisálida y adulto. 


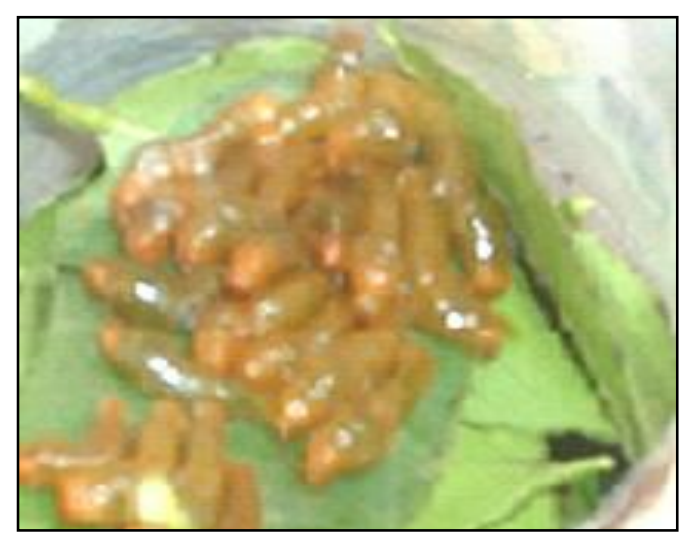

Fig. 5. Orugas de primer instar de Heraclides anchisiades, sus hábitos son gregarios.

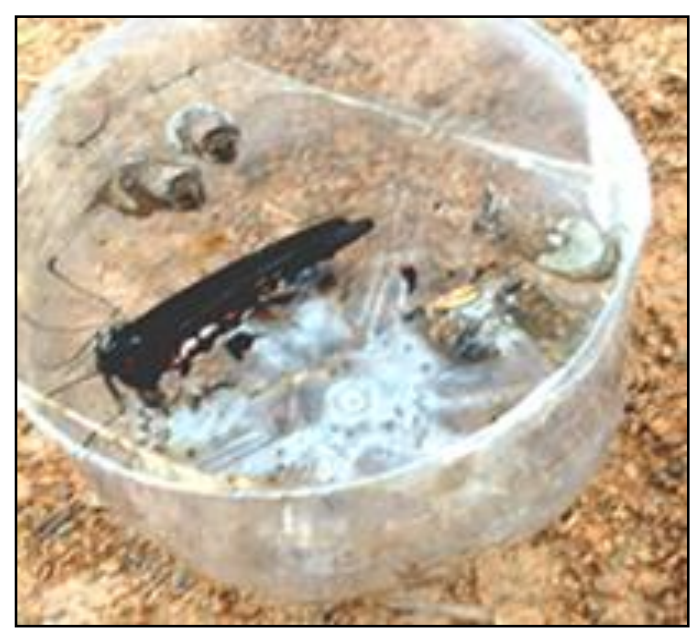

Fig. 7. Adulto, y crisalida de $H$. anchisiades, las pupas parecen una ramita seca

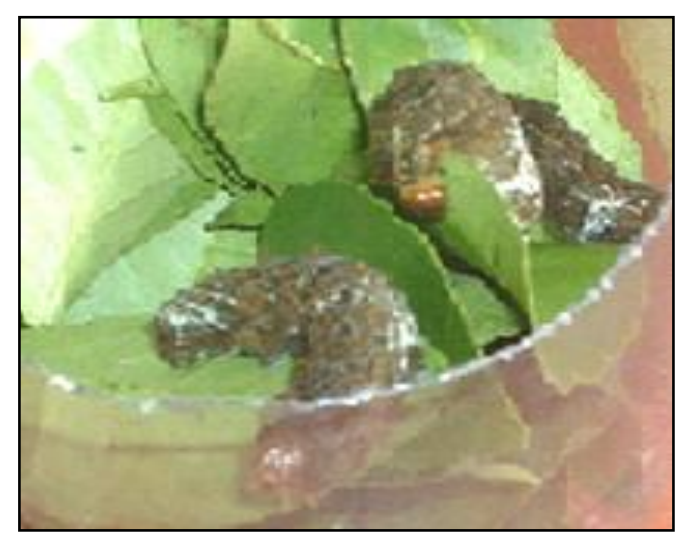

Fig. 6. Orugas de quinto instar, estas orugas se alimentan de Citrus limón.

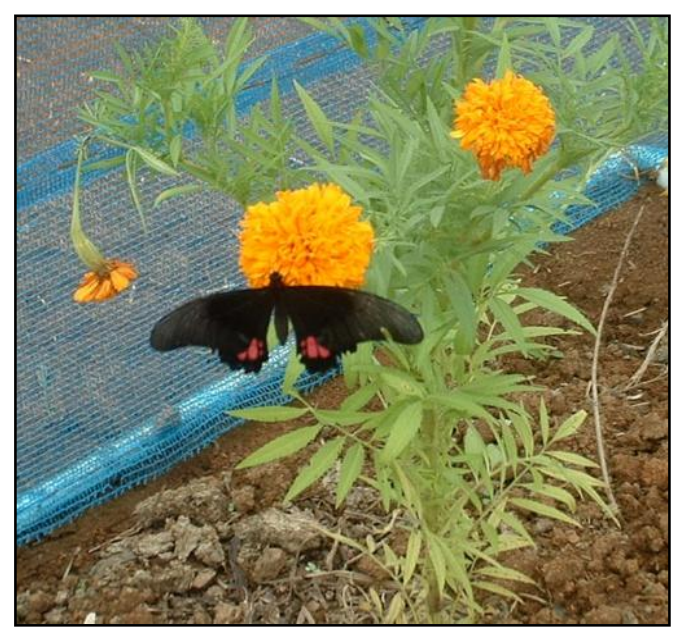

El otro mecanismo de cría empleado fue el rancheo, para implementar este sistema de cría en la escuela se utilizaron envases de botellas plásticas que provenían de los desechos de la comunidad. La zona de cría se situó en los mesones del laboratorio de Biología, sitio al cual los niños tuvieron acceso diariamente, pues fueron ellos quienes asumieron la responsabilidad de la cría por lo que era necesario que alimentaran, limpiaran y observarán los cambios sufridos por las orugas diariamente. Sólo de esta manera fue evidente la procedencia de las mariposas y polillas. Con la implementación de este sistema se tuvo éxito con la cría de $H$. anchisiades, que no fueron las únicas orugas criadas, puesto que también eclosionaron polillas; esto debido a la estrategia empleada, ya que el objetivo principal era enseñar el procedimiento para la cría de orugas e incentivar el aprendizaje por descubrimiento en los estudiantes de la institución, propósito que se cumplió; tanto los niños, como algunos jóvenes aprehendieron, concibiendo que 
aprehender es: relacionar, sinónimo de agarrar, que según el ecologista argentino Grimberg (citado en Wilches, 1996), no es apropiarse de algo si no fusionarse con ello. Con lo anterior se logró que en los niños y niñas quedaran asimilados las experiencias y los descubrimientos realizados por medio y alrededor de la cría. En ningún momento se hizo selección de orugas, puesto que no se quiso desaprovechar el entusiasmo y empeño que los niños y niñas ponían en su búsqueda y captura.

Se realizaron salidas pedagógicas de observación de mariposas a sitios aledaños a la escuela donde se buscó que los estudiantes reflexionaran sobre la importancia de cuidar y preservar zonas de bosque que aún se encuentran en pie y que son albergue de varias especies de plantas y animales. Durante las salidas algunos sitios visitados fueron aprovechados para realizar actividades lúdicas que permitieron que los estudiantes entraran en contacto con espacios naturales y facilitaron procesos de sensibilización en los niños. Estas actividades hacen parte de las estrategias que menciona Caduto, para la enseñanza de valores ambientales, la cual utiliza el enfoque del aprendizaje a través de la experiencia, y se conoce con el nombre de aprendizaje basado en actividades prácticas (Caduto 1996). Las salidas de campo permitieron llevar a los estudiantes fuera del centro escolar, puesto que a través de la experiencia de primera mano, la interacción con la naturaleza y los seres que la conforman se logra el desarrollo de valores; es importante recalcar que las salidas siempre deben estar acompañadas de "procesos de análisis y reflexión", por tal razón se enfatizó sobre la importancia que trae el compromiso de ser responsables directos en la transformación del entorno, y las consecuencias, no solo ambientales, sino culturales y sociales que ello implica. En la población de Las Damas y en especifico en los niños y niñas, se hace evidente la carencia de afecto, en aspectos simples y cotidianos como los saludos, la forma en que se relacionan y tratan; en este sentido los juegos fueron muy importantes ya que fortalecieron la dimensión socio-afectiva, acercando a los niños con el conocimiento de su vereda, permitiendo el reconocimiento de sí mismos, de sus compañeros, padres y docentes como actores principales y fundamentales en la transformación de su realidad. Estas experiencias cobran aún más importancia cuando llevamos la escuela a sitios representativos de la vereda, cuando se cruzan los límites del aula de clase. El reconocimiento por parte de los y las estudiantes de sus recursos, el hacerse conscientes de lo que allí se encuentra, en nuestro caso de las mariposas (el bosque que las resguarda y todos sus componentes bióticos y abióticos), permite que se reconozcan a sí mismos en ese sitio. Las actividades lúdicas de esparcimiento, realizadas en todos y cada uno de los momentos, reafirmaron los hilos de amistad, solidaridad y confianza que se tejen alrededor de la escuela y los procesos que en ella se llevan. Al mismo tiempo, permitió ampliar el campo de estudio, aprovechando los recursos con que la comunidad cuenta.

Con el fin de crear un catálogo donde se encontraran las mariposas presentes en los alrededores de la vereda, se realizaron observaciones y muestreos de mariposas en el Caño Bálsamo, el Nacedero la Cristalina y la Escuela, entre los meses de agosto a octubre de 2007. La siguiente grafica muestra la proporción de familias de mariposas diurnas encontradas en Las Damas de acuerdo al número de especies: 


\title{
Proporción de familias por No. de especies de mariposas encontradas en Las Damas
}

\author{
- Papilionidae $\square$ Pieridae $\square$ Nymphalidae $\square$ Hesperidae
}

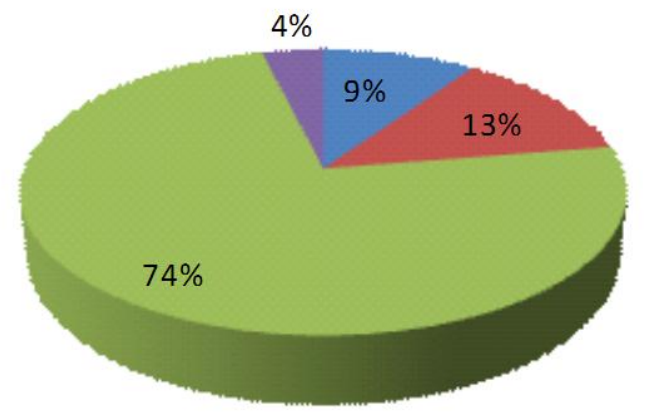

Fig. 9. Proporción de familias de mariposas diurnas encontradas entre los meses de agosto a octubre de 2007 en los sitios cercanos a la IELD.

Se identificaron mariposas pertenecientes a las familias Papilionidae, Nymphalidae, Pieridae y Hesperidae, la familia mas representativa encontrada en Las Damas fue Nymphalidae, con 23 especies identificadas, esto debido a que esta es la familia más diversa de mariposas, con aproximadamente 7250 especies a nivel mundial de las cuales el $42 \%$ son neotropicales (García, Constantino, Heredia y Kattan 2002),el segundo lugar lo ocupó la familia Pieridae, ya que se lograron identificar 4 especies con representantes de dos de las tres subfamilias presentes en el neotropico, Pierinae (Cunizza hirlanda apicalis) y Coliadiane (Phoebis argante larra, Anteos menipe, Phoebis senae marcellina). El sistema de clasificación utilizado para la identificación se basa en el propuesto por Harvey 1987 y Ehrlich 1958. El listado de mariposas encontradas en las Damas se puede observar en la Tabla 3.

Los especímenes fueron fotografiados con el fin de crear un material visual de consulta (catálogo) para que sirviera de referencia para los estudiantes e integrantes de la comunidad.

Tabla 3. Especies de mariposas encontradas en los alrededores de la IELD.

\begin{tabular}{|c|l|l|}
\hline FAMILIA & SUBFAMILIA & \multicolumn{1}{|c|}{ GÉNERO } \\
\hline \multirow{4}{*}{ Papilionidae } & \multirow{2}{*}{ Papilioninae } & Heraclides anchisiades anchisiades (Esper, 1788) \\
\cline { 3 - 3 } & & $\begin{array}{l}\text { Heraclides chiansiades chiansiades (Westwood, } \\
\text { 1872) }\end{array}$ \\
\cline { 3 - 3 } Pieridae & \multirow{3}{*}{ Coliadinae } & Heraclides sp \\
\cline { 3 - 3 } & & Phoebis argante larra (Fabricius, 1775) \\
\cline { 3 - 3 } & & Anteos menipe (Hubner, 1818) \\
\cline { 3 - 3 } & Pierinae & Cunizza hirlanda apicalis (Fruhstorfer, 1907) \\
\hline
\end{tabular}




\begin{tabular}{|c|c|c|}
\hline \multirow{23}{*}{ Nymphalidae } & \multirow{5}{*}{ Nymphalinae } & Anartia amathea (Lineaus 1758) \\
\hline & & Anartia jatrophae ((Linnaeus, 1763) \\
\hline & & Siproeta stelenes (Linnaeus, 1758) \\
\hline & & Junonia evarete (Cramer, 1779) \\
\hline & & Historis acheronta (Fabricius 1775) \\
\hline & Morphinae & Morpho menelaus (Linneaus, 1758) \\
\hline & Apaturinae & Doxocopa Kallina (Staudinger 1886) \\
\hline & \multirow[t]{2}{*}{ Danainae } & Lycorea halia (Hübner, 1825) \\
\hline & & Danaus gillipe gillipe \\
\hline & \multirow{7}{*}{ Bilblidinae } & Hamadryas arinome arinome (Lucas, 1853) \\
\hline & & Panacea regina (Bates, 1864$)$ \\
\hline & & Marpesia berania (Hewitson, 1852) \\
\hline & & Marpesia petreus (Cramer, 1776) \\
\hline & & Nassaea hewitsoni (C. E R. Felder, 1859) \\
\hline & & Eunica taurione \\
\hline & & Eunica clitia \\
\hline & \multirow[t]{2}{*}{ Heliconiane } & Dione juno (Cramer) \\
\hline & & Heliconius \\
\hline & \multirow{5}{*}{ Charaxinae } & Zaretis Isidora \\
\hline & & Fountainea eurhyphile eurhyphile \\
\hline & & Fountainea rhyphea rhyphea Cramer (1775) \\
\hline & & Pseudocahraxeu xenocrates \\
\hline & & Menphis glauce (C. \& R. Felder, 1862) \\
\hline
\end{tabular}

El trabajo con el comité ambiental de la institución conformado por estudiantes de primaria, bachillerato y algunos docentes, permitió generar un espacio de participación considerando que está ligada a los procesos de apropiación de la realidad, que desarrollan los individuos y colectivos de una comunidad (MEN, 2002); la participación permite que las personas y los grupos sociales desarrollen su sentido de responsabilidad y tomen conciencia de la urgente necesidad de prestar atención a los problemas del ambiente, para asegurar que se tomen medidas al respecto (García y Rosales, 2000). El incentivar la participación activa del comité en los procesos relacionados con las problemáticas ambientales de la escuela, utilizando como excusa el estudio de las mariposas, permitió dar inicio a la búsqueda de soluciones sistémicas de las mismas. En estos espacios los estudiantes conscientes de su responsabilidad ambiental propusieron el desarrollo de una serie de actividades encaminadas a fortalecer la acción del comité. Se convocó a todos los integrantes a reuniones; como producto de éstas se generaron propuestas entorno a cuáles debían ser los principales objetivos del comité; entre los cuales se destaca implementar acciones que incentiven actitudes y valores de respeto, responsabilidad y solidaridad por el ambiente, así como fortalecer el PRAE de la institución, de esta forma se trazó una metodología de trabajo para el último periodo del 2007 ver tabla 4. 
Tabla 4. Actividades propuestas para el siguiente periodo académico.

\begin{tabular}{|c|c|}
\hline Actividad & Objetivos \\
\hline $\begin{array}{l}\text { Torneo de } \\
\text { banquitas }\end{array}$ & $\begin{array}{l}\text { - Generar un espacio de participación y sana recreación a los } \\
\text { estudiantes. } \\
\text { - Promover la integración de la comunidad educativa. } \\
\text { - Recolectar fondos para realizar los letreros de señalización } \\
\text { del nacedero la cristalina. }\end{array}$ \\
\hline $\begin{array}{l}\text { Jornada de } \\
\text { limpieza del } \\
\text { Nacedero la } \\
\text { Cristalina }\end{array}$ & $\begin{array}{l}\text { - Proteger y conservar el nacedero la Cristalina, principal } \\
\text { recurso hídrico con que cuenta la institución. } \\
\text { - Incentivar la participación activa de toda la comunidad, y } \\
\text { desarrollar el sentido de apropiación para generar valores } \\
\text { de respeto y responsabilidad hacia la naturaleza. }\end{array}$ \\
\hline $\begin{array}{l}\text { Salidas de } \\
\text { campo }\end{array}$ & $\begin{array}{l}\text { - Generar procesos de reflexión y sensibilización entorno a la } \\
\text { importancia de cuidar y preservar los recursos naturales } \\
\text { con que cuenta la vereda las Damas. } \\
\text { - Identificar y observar las especies de mariposas que se } \\
\text { encuentran en los alrededores de la institución. } \\
\text { - Reconocer la importancia de los recursos naturales y de } \\
\text { proteger el bosque en pie. }\end{array}$ \\
\hline
\end{tabular}

Para cumplir los objetivos propuestos; el comité ambiental decide realizar un cronograma con una serie de actividades, en las cuales participaron los estudiantes de la institución. Estas actividades que partieron de la iniciativa de los estudiantes son de gran importancia puesto que ayudan a los individuos a adquirir valores sociales así como un profundo interés por el ambiente, permitiéndoles una participación activa en la protección y mejoramiento del mismo, por tal razón se aconseja que en los programas de Educación Ambiental EA se incluyan la realización de pequeñas acciones para mejorar la calidad ambiental. Se intenta conseguir con ello un efecto multiplicador, de forma que muchas pequeñas acciones puedan producir grandes resultados, tanto por su efecto acumulador como sinérgico. Sin embargo, no se debe caer en el error de centrarse solo en la realización de este tipo de actividades sin que haya un proceso de reflexión y una evaluación de sus resultados, estas acciones deben ser una constante dentro de la EA.

El PRAE de la Institución Educativa Las Damas esta orientado a la formación de nuevos ciudadanos y ciudadanas éticos frente a la vida y frente al ambiente, responsables en la capacidad para comprender los procesos que determinan la realidad social, natural y cultural respondiendo a las necesidades del entorno donde se desarrolla el educando, de acuerdo a esta situación los profesores de Las Damas han avanzado en el desarrollo del PRAE de la Institución el cual se enfoca en el cuidado y protección del Nacedero la 
Cristalina, uno de los objetivos planteados en el PRAE es fortalecer los valores, la ética y el sentido de pertenencia por la conservación de los recursos naturales, culturales y sociales (con una visión sistémica), este trabajo permitió acercar a los estudiantes un poco más al conocimiento de su vereda. Entrar en contacto con la naturaleza, desarrollar actividades lúdicas y juegos fortalece valores y actitudes en los niños, otro aspecto fundamental es que mediante la implementación de sistemas de cría de mariposas en la escuela se logró sensibilizar a la comunidad acerca de la importancia de cuidar y preservar los recursos ya que son indispensables para el desarrollo de la comunidad, y vitales para la sobrevivencia de otras especies entre ellas las mariposas, también se incentivó la búsqueda de conocimiento . La técnica de evaluación del impacto del proyecto en la comunidad fue de carácter cualitativo, la cual permite valorar la acción educativa y mejorarla durante su desarrollo, atendiendo no solo a los resultados si no sobre todo a los procesos, para evaluar actitudes y valores en los niños se utilizó la observación sistémica, esta observación se puede realizar a través del seguimiento directo de las actividades estructuradas en un plan de trabajo, bien se realicen dentro o fuera del aula (García, J. y Rosales, J., 2000) cuando se realizan salidas de campo, obras de teatro, representaciones artísticas o cualquier otra actividad académica, se puede observar y realizar seguimiento al comportamiento de los individuos y al grupo en general. Otra herramienta implementada para evaluar actitudes y valores, fue a través de charlas informales con los padres de familia, quienes hicieron referencia a cambios de actitudes en sus hijos, ya que observaban valores (respeto) y actitudes (apropiación) en los comportamientos cotidianos y habituales de los niños. Estos cambios en su comportamiento generan casi de manera reciproca, inquietud y curiosidad en los padres, quienes se acercan para descubrir e indagar sobre la razón del cambio, también se puede confrontar a los niños con alguna realidad concreta (situación problema) lo cual deja en evidencia la manera en que el niño o la niña da solución o maneja dicha situación. Otro aspecto relevante es abordar el trabajo interdisciplinario, el cual es entendido como la practica permanente del dialogo entre disciplinas, saberes especialistas y actores sociales -entre otros- (MEN y MMA, 2002), para lo cual se buscó realizar actividades en espacios académicos diferentes a la clase de ciencias, entre los cuales se destaca la realización de una obra de teatro relacionada con el ciclo de vida de las mariposas en la cual los estudiantes fueron los encargados de crear el argumento y el vestuario, lo cual tan solo es una pequeña muestra de alternativas que se pueden realizar para buscar la interdisciplinariedad, sin embargo este proceso debe intentar abarcar todo el currículo, para lo cual es necesario profundizar en el desarrollo del mismo y en el planteamiento de las actividades a desarrollar.

\section{CONCLUSIONES}

Durante la investigación desarrollada en la Institución Educativa Las Damas, la cartografía social fue una herramienta útil, ya que permitió profundizar y conocer las percepciones que presentaban los integrantes de la comunidad con respecto a las mariposas y su entorno, de esta forma se pudo adecuar la metodología al contexto y los intereses específicos de la comunidad.

Los niños y niñas de Las Damas en la apropiación de su entorno tienen como referencia sitios en los cuales entablan relaciones afectivas-cognitivas. Las salidas de campo 
utilizadas como escenarios vivos de aprendizaje en los cuales se realizaron procesos de análisis y reflexión permitieron a los niños, niñas, docentes, reconocerse como actores principales, responsables de la transformación (positiva o negativa), de su entorno, involucrando una mirada sistémica (cultural, social, política y económica).

El trabajo con mariposas permitió despertar la sensibilidad, y la curiosidad en la comunidad Las Damas y de este modo redescubrir su entorno y los seres que lo conforman. Al implementar el estudio de las mariposas como estrategia en la Educación Ambiental se logró poner en marcha procesos organizativos generando una dinámica de trabajo que posibilitó la sensibilización y constante participación de los diferentes actores que conforman la comunidad.

Las salidas de campo a sitios importantes para los estudiantes de Las Damas, permitió fortalecer valores de respeto, responsabilidad y solidaridad, generando sentido de apropiación; primer paso en la incorporación de una Conciencia Ambiental, lo cual es prioritario en la búsqueda de soluciones a problemáticas ambientales particulares de la comunidad.

Las actividades lúdicas realizadas en las visitas a sitios representativos de la vereda y a sitios aledaños a la escuela fueron de gran importancia ya que tanto los juegos como el gozo, son relevantes en el aprendizaje, más aun cuando los que se buscó fue generar en los estudiantes un cambio trascendental de actitud frente a situaciones cotidianas, estos cambios se hicieron evidentes en la forma como los niños interactuaron con su entorno y con sus compañeros.

La participación del Comité Ambiental en los procesos educativos-ambientales de la escuela, así como el desarrollo de actividades por parte de sus integrantes encaminadas al fortalecimiento del mismo, generaron sentido de responsabilidad así como preocupación e interés por las problemáticas locales posibilitando la participación activa y la iniciativa de los estudiantes en la búsqueda de soluciones a dichas problemáticas.

\section{Bibliografía}

- ALVAREZ J.; ALVAREZ , J. 1984. Mariposas diurnas de Venezuela: Introducción a su conocimiento. Caracas Venezuela. Editorial Arte. 199 p.

- CADUTO M. J. 1996. Guía para la enseñanza de valores ambientales. París. UNESCO. p. 106

- DIAZ, J; AVILA, L. 2002. Sondeo del mercado mundial de mariposas. Instituto de Investigaciones de recursos biológicos Alexander Von Humboldt. sitio de internet: http:///..mercados verdes../Mariposas.pdf Fecha de última revisión 26 de mayo de 2007. Fecha de último acceso 11 de abril de 2009.

- GARCÍA, C.; CONSTANTINO L.; Heredia M.; Kattan G. 2002. Guia de campo: Mariposas comunes de la cordillera central de Colombia. Cali, Colombia. Wildlife Conservation Society. 125 p.

- GARCÍA, J.; ROSALES, J. 2000. Estrategias didácticas en Educación Ambiental, Málaga, EDICIONES ALJIBE. $231 \mathrm{p}$.

- GÓMEZ, R. 2006. Plan de manejo propuesto para la cría de mariposas promisorias como alternativa productiva para comunidades indígenas para la Amazonía Colombiana. 
Boletín Sociedad Entomológica Aragonesa (38): 451-460 sitio de internet: http://www.sea-entomologia.org/PDF/GeneraInsectorum/GE-0057.pdf . Fecha de última revisión 22 de marzo de 2008. Fecha de último acceso 10 de Marzo de 2009.

- EHRLICH, P. R. 1958. The comparative morphology, phylogeny and higher classifi cation of the butterflies (Lepidoptera: Papilionoidea). University of Kansas Science Bulletin, 39: 305-364.

- HABEGGER, S; MANCILA, I; 2006. El Poder de la Cartografía Social en las prácticas contrahegemónicas o La Cartografía Social como estrategia para diagnosticar nuestro territorio sitio de internet:

http://areaciega.net/index.php/plain/cartografias/car_tac/el_poder_de la cartografia_soc ial. Fecha de última revisión 22 de marzo de 2008. Fecha de último acceso 10 de Marzo de 2009.

- LE CROM, J.; CONSTANTINO; SALAZAR, J. 2002. Mariposas de Colombia. Tomo I. Bogotá. Carlec. 112. p.

- Ministerio de Educación Nacional. 2002. Política Nacional de Educación Ambiental Sina. Colombia: Autor.

- Ministerio de Educación Nacional y Ministerio del Medio Ambiente. 2002. Reflexión y Acción: el Dialogo Fundamental para la Educación Ambiental. Colombia: Autores.

- MOLINA. 2002, Ciclos Biológicos de Especies diurnas de Lepidoptera del Bosque Seco Tropical, Guayaquil, Ecuador.

- MOTTA, J. A. 1998. La lúdica, procedimiento pedagógico. Bogotá. Universidad Nacional. $26 \mathrm{p}$.

- RODRÍGUEZ, M. 1998. Mito, identidad y rito. Mexicanos y chicanos en California. México. CIESAS. p. 253

- VÉLEZ, J; SALAZAR, J. 1991. Mariposas de Colombia. Bogotá. Villegas Editores. 167 p.

- WILCHES, G.1996 La letra con risa entra. Colombia. Ecofondo Dupligráficos. 193 p. 